\title{
AVALIAÇÃO DISCENTE DO MÉTODO PBL APLICADO NO CURSO DE ENGENHARIA DE COMPUTAÇÃO DA UEFS: UMA ANÁLISE LONGITUDINAL
}

\author{
Carlos Alberto dos Santos da Silva ${ }^{1}$; David Moises Barreto dos Santos \\ 1. Bolsista FAPESB, Graduando em Engenharia de Computação, \\ Universidade Estadual de Feira de Santana, e-mail: carlosadsds@gmail.com \\ 2. Orientador, Departamento de Ciências Exatas, \\ Universidade Estadual de Feira de Santana, e-mail: davidmbs@uefs.br
}

PALAVRAS-CHAVE: PBL, Engenharia de Computação, Estudo Longitudinal.

\section{INTRODUÇÃO}

O método PBL (Problem-Based Learning), usado no curso de Engenharia de Computação da UEFS, tem como finalidade fazer do aluno o principal ator do seu processo de aprendizagem, enquanto o professor (tutor) assume a responsabilidade de acompanhar tal processo (Duch, Groh, \& Allen, 2001). Apesar de o curso funcionar desde 2003 e muitos aspectos do PBL já terem sido lapidados, ainda existe uma carência de uma avaliação geral do método. Os autores deste relatório realizaram uma avaliação anteriormente, através de método quantitativo (usando questionário), da percepção dos estudantes sobre a implementação do PBL no referido curso, entre o final de 2014 e início de 2015 (Santos \& Silva, 2015; Silva \& Santos, 2015). Uma outra avaliação foi realizada em 2016. Porém, sozinhos, os resultados destas pesquisas apenas oferecem um retrato pontual e estanque da realidade. Neste contexto, faz-se mister uma avaliação contínua do método PBL sob a ótica discente, sobretudo tomando a seguinte questão de pesquisa como norte: qual a percepção que o graduando de Engenharia de Computação tem acerca do PBL ao longo do tempo? O destaque aqui é investigar se existe uma variação da percepção ao longo do tempo e quais fatores influenciam em tal percepção. Para realizar essa avaliação, utilizouse a pesquisa longitudinal, método de investigação em que pessoas de um mesmo grupo são estudadas em diferentes épocas de suas vidas, em um dado período no tempo, para investigar mudanças (Boyd \& Bee, 2011; Hochman et al., 2005).

\section{METODOLOGIA}

SUJEITOS: 161 matriculados no curso de Engenharia de Computação no semestre 2017.2 (das quais 63 pessoas também haviam participado da pesquisa em 2016.1). Os 63 estudantes tiveram média de idade de 21 anos (desvio padrão $\cong 1,86$ ), enquanto 53 eram homens (84\%), 10 eram mulheres (16\%). INSTRUMENTO: Q-PBL, um questionário que em suma, avalia 17 itens relativos a aspectos específicos da dinâmica própria do PBL (motivação, sessão tutorial, grupo tutorial, problema, tutor, avaliação e feedback, adaptação) e a percepção de 9 habilidades melhoradas a partir deste método. Ademais, existem mais duas questões: uma para realizar uma avaliação geral do método PBL, com escala de 10 pontos, e outra para tecer comentários livres. Assim, totaliza-se 28 itens. PROCEDIMENTO: A coleta aconteceu presencialmente mediante visitas às salas de aulas nos meses de dezembro de 2017 e fevereiro de 2018. É importante destacar que esta pesquisa foi aprovada pelo Comitê de Ética em Pesquisa com Seres Humanos (CAAE $\mathrm{n}^{\mathrm{o}}$ 31747814.8.0000.0053). ANÁLISE: A análise destes dados se deu através de estatística descritiva e inferencial, com suporte eletrônico do software SPSS 20. 


\section{RESULTADOS E/OU DISCUSSÃO}

Na primeira parte do questionário foram avaliados os Aspectos Específicos do PBL. Ao comparar as médias registradas pelos estudantes durante as duas coletas, pôde-se observar que durante o semestre 2016.1, todos os itens do questionário foram melhor avaliados do que no semestre 2017.2. Apesar de boa parte das questões terem apresentado médias próximas, houve uma notável diminuição nas pontuações atribuídas pelos estudantes. Na primeira coleta, por exemplo, apenas a questão referente a clareza dos problemas obteve média abaixo de 4 pontos, enquanto na segunda, quatro itens ficaram abaixo desse valor. Também é possível notar uma redução nas médias das questões relacionadas ao tutor, no fornecimento de feedbacks ao grupo tutorial $(-27,6 \%)$ e na clareza ao expor os critérios de avaliação do produto $(-21,7 \%)$.

A Fig. 1 representa graficamente as médias para cada questão dos aspectos específicos do PBL e evidenciam a diminuição da pontuação para todas questões, com destaque para a queda na avaliação das questões 11 e 12, referentes ao tutor.

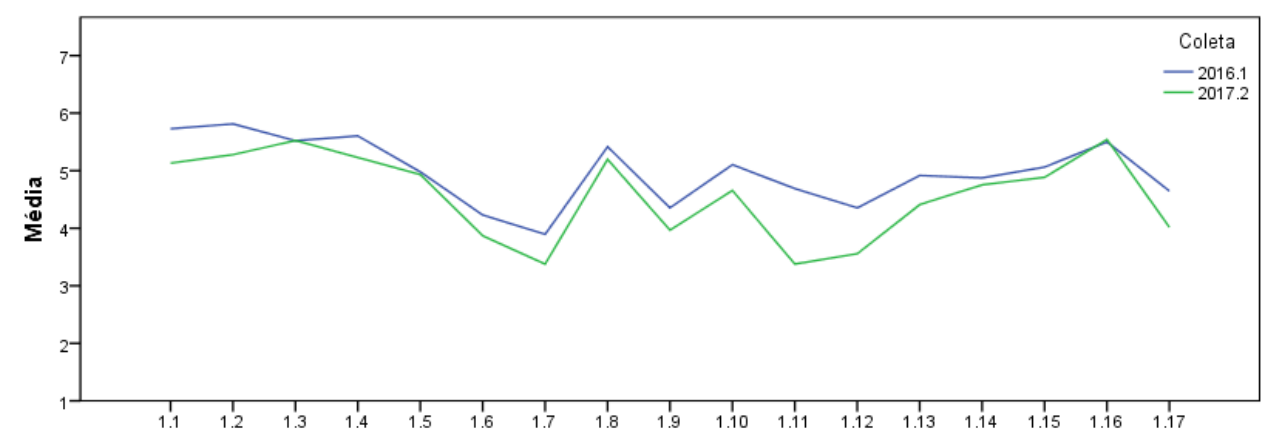

Fig. 1. Comparação entre as coletas 2016.1 e 2017.2, para os aspectos específicos do PBL.

A redução nas médias das questões não atingiu apenas os aspectos específicos do PBL, mas também as questões relacionadas às habilidades percebidas pelos estudantes. Apesar disso, os itens referentes ao autodidatismo e a resolução de problemas permaneceram sendo aquelas com as melhores avaliações dos participantes. Em contrapartida, o planejamento, que era uma das habilidades mais bem avaliadas tornouse a segunda menos bem avaliada, conforme ilustrado no gráfico da Fig. 2.

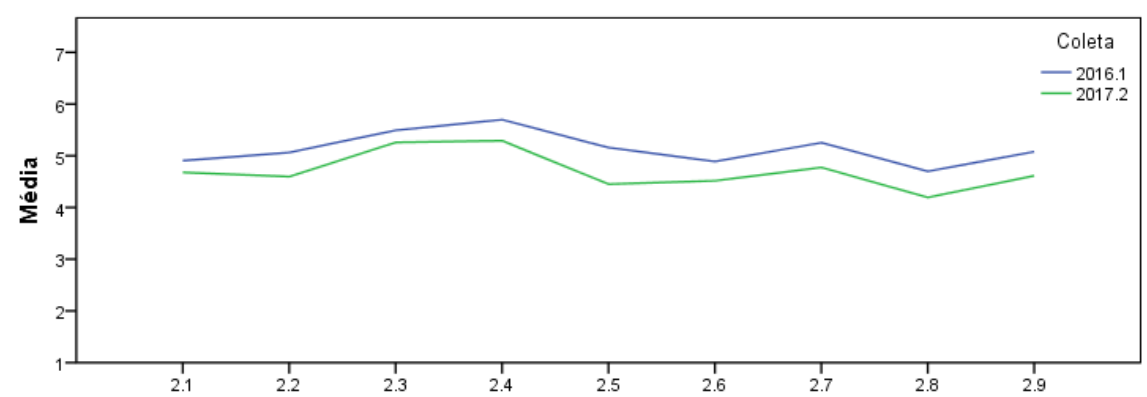

Fig. 2. Comparação entre as coletas 2016.1 e 2017.2, para as habilidades desenvolvidas.

No questionário havia ainda um item que avaliava a satisfação dos estudantes com o método PBL aplicado no curso de Engenharia de Computação. Enquanto na primeira coleta fora registrada uma média de 6,9 (desvio padrão $=1,991$ ), na segunda coleta essa pontuação caiu $17,3 \%$, registrando média de 5,7 (desvio padrão $=2,217$ ). Isso representa uma diminuição considerável na satisfação dos participantes com o PBL. 
Os entrevistados foram agrupados em seis categorias, que os caracterizavam como: calouros, não calouros, repetentes, não repetentes, masculino e feminino. Assim, através do Paired Samples T-Test avaliamos cada um desses grupos de estudantes em dois momentos diferentes no tempo; ora no semestre 2016.1, ora no semestre 2017.2. Por exemplo, analisou-se a opinião dos calouros na primeira e segunda coleta (lembrando que nesta segunda coleta já não eram, com efeito, calouros). A percepção no declínio das médias de avaliação foi maior para os estudantes do sexo masculino (Fig. 3), os não calouros (Fig. 4) e os que nunca haviam repetido um MI (Fig. 5).

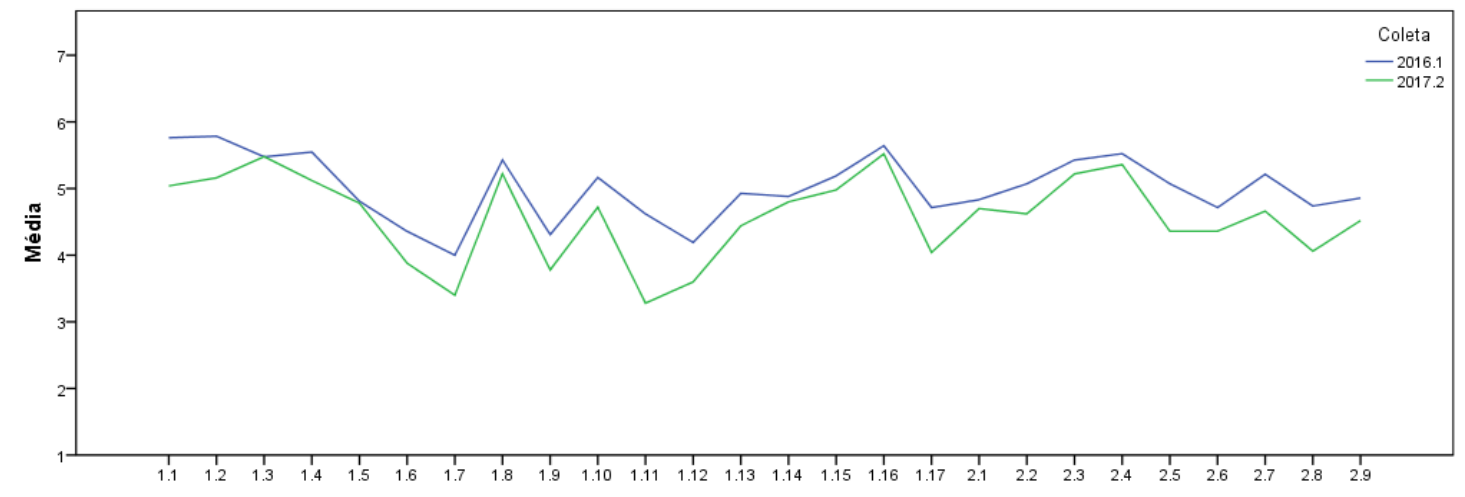

Fig. 3: Percepção dos estudantes do sexo masculino entre as 2 coletas.

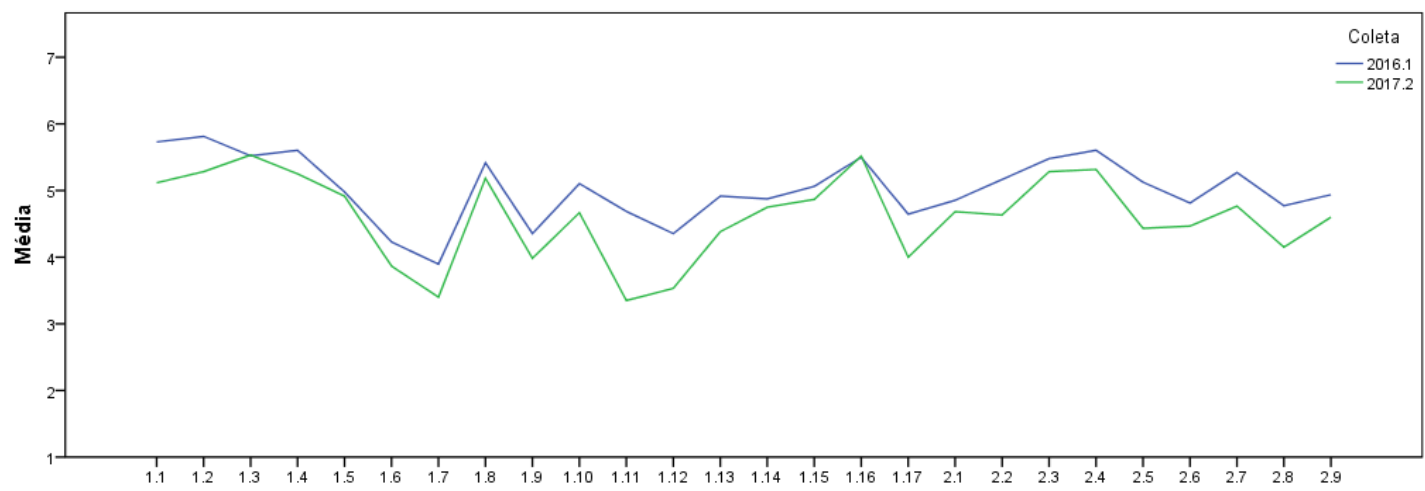

Fig. 4: Percepção dos não calouros entre as 2 coletas.

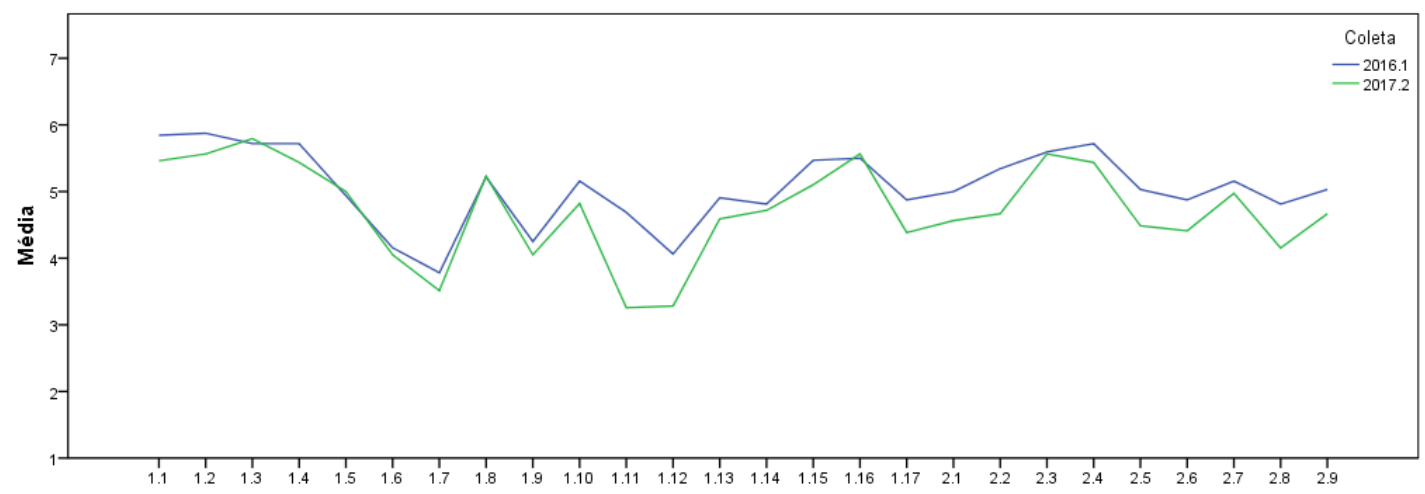

Fig. 5: Percepção dos estudantes não repetentes entre as 2 coletas.

Existem dois tipos de variáveis independentes: entre-sujeitos (Between-Subjects Factors - grupos independentes, como maturidade, repetência ou sexo) e intra-sujeitos (WithinSubjects Factors - grupos relacionados com medidas repetidas, a exemplo do tempo). Para comparar as diferenças médias entre grupos que foram divididos em duas variáveis independentes utilizamos o Two-way Mixed ANOVA. 
Não houve tanta diferença na interação entre o tempo e os grupos, exceto por algumas questões em particular (6 para tempo*maturidade e 2 para tempo*sexo). Também houve pouca diferença estatisticamente significativa entre os grupos (4 para maturidade, 6 para repetência e 1 para sexo). Com efeito, a percepção dos entrevistados apresentou mais mudanças ao longo do tempo.

\section{CONSIDERAÇÕES FINAIS}

Os primeiros resultados do estudo mostraram um declínio na avaliação dos entrevistados, percebido em maior escala por estudantes do sexo masculino, não calouros e não repetentes. Os estudantes permanecem vendo o PBL como um motivador para a busca da própria aprendizagem e as sessões tutoriais como catalisadoras na resolução dos problemas. $\mathrm{O}$ autodidatismo e a resolução de problemas permanecem como as habilidades melhor desenvolvidas pelo PBL. A avaliação dos estudantes quanto aos feedbacks fornecidos pelos tutores e a clareza dos critérios para a avaliação do produto, entretanto, foram as questões com os maiores decréscimos de médias entre as coletas, revelando um aumento da insatisfação dos estudantes quanto a esses pontos. A percepção dos estudantes parece vir mudando com o tempo, especialmente nas comparações realizadas entre entrevistados iniciantes e veteranos no curso.

Apesar de proporcionar uma noção inicial de como os estudantes tem percebido o PBL no curso ao longo de dois semestres, é necessário mais do que dois pontos no tempo para apontar as causas e efeitos da avaliação dos entrevistados. Nesse sentido, como trabalhos futuros, pretende-se realizar novas coletas para inferir novamente a opinião dos estudantes sobre o PBL aplicado no curso, visando construir uma base de dados cada vez maior e mais consistente, de maneira a propor mudanças pontuais na forma como o método é colocado em prática.

\section{REFERÊNCIAS}

BOYD, D., \& BEE, H. (2011). A criança em crescimento (1st ed.). Porto Alegre: Grupo A - Artmed.

DUCH, B. J., GROH, S. E., \& ALLEN, D. E. (2001). Why Problem-Based Learning? A case study of institutional change in undergraduate education. In The power of problembased learning: a practical "how to" for teaching undergraduate courses in any discipline (pp. 3-12). Loudoun County: Stylus Pub.

HOCHMAN, B., NAHAS, F. X., FILHO, R. S. de O., \& FERREIRA, L. M. (2005).

Desenhos de pesquisa. Acta Cirúrgica Brasileira, 20, 2-9.

SANTOS, D. M. B., \& SILVA, C. A. S. (2015). Evaluation of students' general perceptions of Problem-Based Learning in a Computer Engineering program in Brazil. Problems of Education in the 21st Century, 68, 74-83. Retrieved from http://www.scientiasocialis.lt/pec/?q=node/996

SILVA, C. A. S., \& SANTOS, D. M. B. (2015). Avaliação do método PBL por calouros do curso de Engenharia de Computação da UEFS. Anais Do XLIII Congresso Brasileiro de Educação Em Engenharia. 